\title{
Pour une pédagogie de la créativité en classe de langue Réflexion théorique et pratique sur la triade créativité, émotion, cognition
}

Isabelle Puozzo Capron, Haute Ecole Pédagogique du Canton de Vaud, Suisse

La recherche sur la créativité est devenue un enjeu, souvent central, dans de nombreux domaines. À l'école, ce concept se décline différemment d'une discipline à l'autre avec des enjeux qui dépendent des objectifs d'apprentissage. La discipline de référence demeure celle des arts où la production même d'une œuvre est censée impliquer un processus créatif. Cependant, comment l'enseignant de langue peut-il structurer sa planification et construire des tâches qui permettent aussi de développer la créativité de ses élèves ? Quels sont les enjeux pédagogiques et didactiques de la créativité dans le processus d'enseignement/apprentissage de la langue? Dans quelle mesure la créativité peut-elle favoriser l'apprentissage?

Cet article propose une définition du concept de la créativité en langue à partir de plusieurs cadres théoriques comme la psychologie, la psychologie de la créativité ou bien encore le domaine artistique. Il s'agit de proposer une définition holistique de la créativité dans le but de construire une pédagogie de la créativité en classe de langue à partir de l'imbrication de références théoriques hétérogènes, mais complémentaires. Une rechercheaction est enfin présentée pour tisser l'articulation entre théorie et pratique et avoir une posture réflexive sur ce qui est potentiellement observable et ce qui mériterait d'être approfondi par des recherches supplémentaires.

\section{1- Pourquoi envisager une pédagogie de la créativité dans l'apprentissage des langues ?}

L'école est le lieu où l'émotion peut constituer « une entrave ou au contraire une aide forte à l'acquisition » (Cuq 80). Il s'agit alors de "parvenir à maitriser ses émotions sans pour autant les supprimer » (80). L'émotion devient ainsi une variable non négligeable du processus d'apprentissage et de la réussite scolaire. En classe de langue, les élèves qui ont déjà des difficultés, souvent dans les activités langagières de production, se retrouvent pénalisés, notamment en contexte d'évaluation. Ces émotions sont des éléments qui parasitent la performance. La problématique de la recherche est de se demander comment favoriser un état émotionnel positif afin d'augmenter la qualité de la performance. Comment créer des conditions didactiques et pédagogiques qui permettent à l'élève de tempérer les émotions qui parasitent sa performance ? 
Ce sont les travaux du psychologue A. Bandura sur le sentiment d'efficacité personnelle [désormais SEP] qui constituent un point de départ pour réfléchir sur l'état émotionnel des élèves. En effet, selon la théorie du SEP, l'une des variables de la réussite scolaire est la perception préalable que l'apprenant a de ses compétences et qui va lui permettre de s'engager ou non dans une tâche : plus la perception de ses compétences est élevée, plus la réussite de sa performance est probable. Bandura ajoute que cette perception est influencée par les états physiologiques et émotionnels. Généralement, plus l'individu perçoit les compétences qu'il maitrise pour atteindre un but, plus les émotions et les états physiologiques sont positifs et inversement. Cette source est donc auto-évaluative car ce sont les élèves qui analysent ces changements du corps et de l'esprit et qui vont leur en donner une interprétation, souvent basée sur des expériences antérieures. Même si cet état émotionnel est une source intrapersonnelle sur laquelle l'enseignant n'agit pas, il peut toutefois s'interroger sur comment construire un environnement d'apprentissage qui favorise un état émotionnel positif chez les élèves.

De plus, en classe de langue, l'évaluation et la production orale sont des situations potentiellement anxiogènes (Piccardo et Puozzo Capron) pour certains élèves. Pourtant, il est impossible d'en faire abstraction. De la même manière que l'enseignant est obligé d'évaluer, il est inconcevable de ne pas travailler la production orale. Il s'agit alors de réfléchir sur des modalités de tâches qui réduisent ces émotions parasites. La piste présentée dans cet article est celle de la pédagogie de la créativité. L'objectif est d'amener les apprenants à faire émerger des émotions facilitatrices d'apprentissage qui tempèrent ainsi leurs émotions parasites liées au contexte de la production orale grâce à la réalisation d'un support créatif.

\section{2- Entre adaptation et nouveauté : pour une définition de la créativité}

«Je crée, donc je suis » (Seely Brown cité par Gardner 95). Cette citation d'un spécialiste de l'entreprise permet de mettre en évidence le déplacement des caractéristiques personnelles attendues chez l'employé d'aujourd'hui qui n'est plus uniquement un professionnel compétent, mais un professionnel qui se doit d'innover pour s'adapter aux besoins de la société. Être créatif implique de chercher à développer ses compétences pour s'adapter à l'évolution de la société (Robinson) et/ou la provoquer. Ainsi, la créativité est devenue un critère d'évolution sociale, lequel a aujourd'hui des répercussions sur les finalités de l'école. Développer la créativité des élèves favorise cette capacité, au sens psychologique du terme, à s'adapter aux changements de l'environnement. Dans une société de plus en plus complexe, les élèves sont censés apprendre à développer entre les murs de l'école cette 
capacité nécessaire à leur vie professionnelle (Robinson). Alors, qu'est-ce que la créativité ? Comment un enseignement structuré peut-il permettre à l'apprenant de développer sa créativité ? Selon T. Lubart, la créativité serait « la capacité à réaliser une production qui soit à la fois nouvelle et adaptée au contexte dans lequel elle se manifeste » (10). Ce degré de nouveauté peut varier, allant d'une «déviation minime » à « une innovation importante » (10). L'adaptation implique que la créativité ne relève pas uniquement d'une liberté absolue et démesurée, mais qu'elle réponde surtout à d'éventuelles contraintes stabilisées par l'environnement et notamment l'enseignant. Pour le contexte scolaire, ce concept confère à la créativité la valeur d'un processus et d'un acte construits et réfléchis. Il ne s'agit pas de laisser créer tout et n'importe quoi dans l'objectif de développer un prétendu génie créateur. Contrairement aux idées reçues, en situation d'apprentissage, la capacité d'être créatif se développe par et grâce à un apprentissage ciblé (Didier et Leuba). La conception même d'un objet est un acte créatif car elle met l'apprenant dans une telle posture qu'il doit faire preuve d'originalité, même minime, et répondre aux contraintes que l'enseignant lui a fixées.

\section{3- Pour une pédagogie de la créativité}

\section{1- Créativité, émotion et cognition : une triade significative pour apprendre}

Les concepts de créativité et d'émotion sont deux objets de recherche sur lesquels se penchent de nombreuses disciplines, parfois en interrogeant le continuum qui les lie, parfois en les analysant séparément ou avec d'autres. La didactique des langues s'intéresse aussi, mais souvent de manière séparée, à ces deux objets de recherche. Cet article propose une réflexion qui articule les concepts de créativité, d'émotion et d'apprentissage de la langue.

Si l'on part d'une définition simple de l'émotion comme «sensation (agréable ou désagréable) », prise dans Le Petit Robert (2002: 865), l'on réduit au minimum un phénomène tellement complexe et il est alors nécessaire de s'appuyer sur différentes disciplines pour le définir et montrer ainsi une partie de cette complexité.

Du côté de la psychologie de la créativité, Lubart définit aussi l'état émotionnel comme un stade court et « intense » (48) qui est la réaction à un stimulus externe. Dans le cadre de la pédagogie de la créativité, ce stimulus serait un objet créé par un apprenant. Cette démarche est nommée par Lubart comme expérimentale dans le sens où l'on s'intéresse aux «effets d'un état émotionnel [...] positif ou négatif (joie, tristesse, surprise, etc.) sur la performance à des tâches de créativité ». « L'intérêt est alors d'observer et d'interpréter les effets facilitateurs ou perturbateurs d'une émotion sur la créativité » (49). Toutefois, à la différence de Lubart qui envisage un continuum entre émotions et créativité, cet article 
interroge plutôt le continuum créativité/émotion dans le contexte de classe de langue en se référant à un autre cadre théorique, celui du domaine de l'art.

En effet, observer une œuvre d'art suscite des émotions dites esthétiques (Deforge). En ce qui concerne l'émotion esthétique suscitée par une œuvre, A. Holley affirme que le « premier niveau de plaisir » est provoqué par les «constituants élémentaires de l'œuvre» (27) tels que la couleur pour une œuvre ou le timbre pour la musique. Vient ensuite la «composante cognitive» apportée par celui qui contemple l'œuvre. Cette composante cognitive qui « nourrit» le «plaisir esthétique » se définit en termes de « connaissances, de références, de comparaisons» (28). Ce lien triadique que Holley tisse entre l'œuvre, l'émotion et la cognition peut être transposé à la pédagogique de la créativité en classe. Les objets créatifs suscitent ce plaisir esthétique par le choix des couleurs, de la matière ou de la mise en forme et la dimension cognitive est d'identifier le contenu disciplinaire dans l'œuvre créative. Toutefois, pour combien d'élèves cette réflexion cognitive se réfère-t-elle à l'objet d'apprentissage sur le texte descriptif et la création d'effets poétiques ? Cette cognition restet-elle sur l'analyse de l'objet d'apprentissage ou bien s'articule-t-elle entre objet créatif et objet d'apprentissage ? Un travail de recherche sur l'activité serait ici nécessaire.

\section{2- En quoi la pédagogie de la créativité favorise-t-elle l'apprentissage?}

Par l'étude des cinq formes d'intelligence (l'esprit discipliné, de synthèse, créatif, respectueux et éthique), H. Gardner affirme que «seul le perfectionnement d'une discipline pouvait donner naissance à des options réellement créatives » (104). Cela signifie qu'il est nécessaire de maitriser des contenus fondamentaux pour être créatif. Si un enseignant demande à l'élève de construire un bateau, les différentes tentatives lui permettront au mieux de faire des hypothèses, mais ne lui permettront pas de comprendre le concept scientifique de flottabilité. Par contre, la construction de ce même bateau, à l'issue de l'apprentissage, pourra mettre en évidence s'il y a eu ou non apprentissage dans la mesure où l'élève sera en mesure d'expliquer comment il s'y est pris pour faire flotter son bateau. Cette métaréflexion à partir du bateau mettra ainsi en évidence l'articulation entre objet d'apprentissage (la flottabilité) et matérialisation dans un support créatif. Cela implique qu'un enseignant qui souhaite pratiquer la pédagogie de la créativité doit, de prime abord, réfléchir sur les contenus disciplinaires (ou autres) fondamentaux à maitriser pour réaliser une tâche créative. M. Csikszentmihalyi et Gardner se rejoignent sur l'idée d'une maitrise préalable de connaissances, pour le premier, d'une discipline pour le second. Csikszentmihalyi donne l'exemple de la construction d'un avion: « un inventeur doit connaitre la physique, l'aérodynamique et les raisons pour lesquelles les oiseaux ne tombent pas du ciel » (14). Une pédagogie de la créativité en classe 
se situe bien à ce même niveau : pour réaliser une performance (qu'elle soit traditionnelle ou créative), l'élève est censé maitriser des savoirs et des capacités et les mobiliser pertinemment (Bourguignon).

L'appropriation de la connaissance implique une certaine persévérance avec des enjeux cognitifs qui se complexifient au fur et à mesure que l'élève construit son objet et apprend. Prenons l'exemple de la métaphore dans la séquence didactique sur le texte descriptif. L'un des critères (contrainte au sens de Lubart) était d'insérer une métaphore si possible visible à l'intérieur de l'objet et obligatoirement dans la production orale. Devoir créer une métaphore implique que l'élève doit, dans un premier temps, comprendre le principe théorique sur la production d'un effet poétique. Puis, il transpose ce principe dans l'objet créatif et dans sa production linguistique. Ceci implique qu'il entre dans une démarche de production, donc dans le processus d'assimilation d'une connaissance procédurale (le fait de créer un effet esthétique adapté à son objet et à son texte), puis éventuellement, dans le processus d'évaluation pour mesurer l'adéquation entre le produit fini et le critère attendu par l'enseignant. Si l'enseignant donne un devoir sur table où il s'agit de relever les métaphores, la tâche montre un apprentissage de la connaissance déclarative, mais pas procédurale. Bien sûr que l'objet créatif n'est pas obligatoire pour effectuer ce passage, ce qui est beaucoup plus significatif dans l'appropriation de l'objet d'apprentissage, c'est la dimension émotionnelle et une approche différente. La créativité devient alors la médiatrice entre les savoirs et la production de l'élève favorisant ainsi à la fois l'acquisition de connaissances et de capacités et le développement d'une compétence qui se manifeste par le biais d'une performance. Ce qui sera beaucoup plus significatif dans l'appropriation de ces connaissances procédurales, ce sera ainsi la dimension émotionnelle et une approche différente à l'apprentissage. En effet, on décentre l'attention de l'apprenant de la dimension linguistique pour la focaliser temporairement sur le support créatif. L'expression de l'émotion, qu'elle prenne la forme d'un rêve, d'un fantasme ou d'une peur, passe par la dimension linguistique et le support créatif est le moyen sur lequel l'apprenant s'appuie pour s'exprimer. L'apprentissage perdure plus longtemps quand on se l'approprie. Si les élèves s'investissent dans l'activité, l'objet créatif rend à son tour l'objet d'apprentissage concret, palpable et visuel. 


\section{4- Recherche-action}

\section{1- Problématique et contexte de la recherche}

Nous venons de voir que la créativité peut agir au niveau cognitif de l'apprentissage et émotionnel, ce dernier permettant de renforcer la dimension précédente. La problématique de la recherche présentée est la suivante : comment la créativité favorise l'apprentissage ? Quel type d'environnement l'enseignant peut-il construire pour que les émotions constituent un levier pour l'apprentissage et non pas un frein ?

L'échantillon se compose de 43 élèves de première année dans un lycée professionnel en Vallée d'Aoste dans le nord de l'Italie. La discipline choisie est le français langue seconde/étrangère. Dans cette région institutionnellement bilingue, où le français est enseigné dans l'idéologie du native speaker, l'enseignement relève cependant plus de la langue seconde, voire étrangère dans certains contextes (Cavalli et al.). L'observation a duré une année (de septembre 2008 à juin 2009). Cette recherche empirique permettait de tester une voie possible d'action didactique favorisant l'émergence d'émotions facilitatrices de l'apprentissage à travers la démarche créative. L'enquête menée dans le cadre de cette recherche s'inscrit dans une typologie de type qualitatif, notamment celle d'une enquête de terrain (Beaud et Weber) et une recherche-action (Becker). Le socio-cognitivisme, plus exactement le sentiment d'efficacité personnelle (Bandura) a constitué l'outil théorique pour élaborer les tâches et les analyser ensuite (Barbier). Cet article se focalise uniquement sur une évaluation sommative construite autour de la créativité. Cette évaluation s'insère dans la séquence d'apprentissage sur le texte descriptif. A la fin de la séquence, les élèves étaient censés rédiger la description de l'hôtel, du restaurant ou bar de leurs rêves, l'exposer ensuite à la classe en présentant un support créatif (l'objet) permettant d'accompagner et de suivre la description (Puozzo Capron; Puozzo Capron et Barioni). Le thème était donc en lien avec leur formation professionnelle. L'idée sous-jacente est que cet objet créatif permet à l'élève de développer son imagination et de s'appuyer sur un support qu'il a lui-même construit afin de stimuler un état émotionnel qui lui permettrait de se percevoir plus auto-efficace. Pour l'élève, il s'agit uniquement de passer du savoir acquis avec l'enseignant (portrait, description physique et caractère, description objective et subjective des personnes, objets et lieux, figures de rhétorique, temps verbaux de l'indicatif, etc.) à la compétence pragmatique (CECR, 2001) de structuration du texte descriptif.

L'hypothèse de départ était de canaliser les émotions parasites de l'apprentissage par l'évocation d'émotions personnelles, en lien avec la formation professionnelle, et d'améliorer ainsi la performance orale. Cette amélioration devait se traduire par : 
- une augmentation du temps de production. Vu que c'était la deuxième évaluation créative de l'année, il s'agissait de passer de 10 à 15 minutes de production orale continue ;

- une maitrise des connaissances et des capacités sur le texte descriptif, manifestée à travers une performance de nature créative permettant la réussite d'un plus grand nombre possible d'élèves.

\section{2-Bilan de l'expérience}

L'une des hypothèses de départ de cette recherche était d'utiliser la créativité pour augmenter le temps de la production orale, et ce à partir de la réalisation de deux projets créatifs : la production orale du premier était de dix à douze minutes et celle du second projet de quinze minutes. Dans ce dernier, l'hypothèse a été validée dans la mesure où de nombreux élèves ont atteint les objectifs fixés et une bonne partie les ayant même dépassés. En effet, plus le support créatif était riche et complexe, plus le temps de la production orale augmentait. Par exemple, un élève a choisi de réaliser un support virtuel avec le jeu des SIMS et a réalisé un monologue continu pendant trente minutes. La production longue correspond au niveau B2 du Cadre Européen Commun de Référence pour les Langues (2001). L'élève a conçu virtuellement l'hôtel de ses rêves avec comme thème "une chambre, un fantasme ». Il y avait la chambre de l'horreur où les lits étaient des cercueils, la chambre des amoureux de l'art avec des statues et chefs-d'œuvre de la Renaissance, la chambre disco avec un parterre multicolore, etc.

Les performances linguistiques se sont révélées d'un niveau supérieur aussi bien quantitativement que qualitativement pour ceux qui ont complexifié leur support (une maquette plutôt qu'un panneau d'affichage). Ce premier objectif a également eu des répercussions sur la dimension pédagogique puisque les élèves ont réalisé qu'ils se dépassaient et qu'ils se mettaient encore plus en jeu que d'habitude, en tout cas plus que si l'on leur avait demandé un exposé traditionnel. Selon l'approche socio-cognitiviste (Bandura) évoquée dans le cadre théorique, il est possible de noter que laisser un espace de liberté où les élèves peuvent choisir de créer un support, implique que ceux-ci doivent penser aux compétences qu'ils maitrisent et qui vont leur permettre de produire un support riche et complexe. L'enseignant s'appuie sur le sentiment d'efficacité personnelle des élèves en valorisant ce sur quoi ils sont compétents. Je parle ici de valorisation et non pas d'évaluation. Cette ostension de l'objet vise à accompagner la description des élèves afin de leur permettre de parler de ce dont ils rêvent. L'évocation même d'un fait agréable a pour objectif de stimuler une émotion facilitatrice de la performance. Le support joue un rôle d'étayage, au 
sens brunerien, pour permettre aux élèves d'avoir un peu plus d'éléments à décrire. D'où la nécessité de valoriser la construction d'un support complexe. Ainsi, la perception d'avoir élaboré un support en fonction de ses compétences vise à permettre à l'élève de percevoir un état émotionnel serein afin d'augmenter à son tour la perception de son auto-efficacité dans sa description et d'obtenir une performance d'une qualité supérieure.

Ces élèves italiens n'ont pas été gênés par le décloisonnement des compétences «autres» puisque de nombreux élèves ont su mettre en valeur leur compétence technologique (jeu des SIMS, PowerPoint, le programme d'Ikea et Planningwiz.com) ou leur compétence manuelle (maquettes réalisées avec du polystyrène, du bois, du carton, du papier, etc.). C'est en analysant les travaux, en écoutant les productions et en connaissant les apprenants de la formation professionnelle que j'ai constaté que ces exposés avaient été meilleurs car ils parlaient de ce qu'ils aimaient et de ce dont ils rêvaient. Voici deux extraits qui mettent en évidence la dimension du rêve. Claudio a réalisé une maquette en bois avec une manivelle pour monter les voitures dans l'hôtel afin de se rendre au drive-in sur le toit ou dormir avec sa Ferrari. Marco a réalisé une maquette avec des Legos et des tissus pour son hôtel cinq étoiles.

Claudio: dans le chose pour monter au drive-in on fait comme ça, ça commence à monter. Arrive ici, et la machine arrive. Là ici, si vous pouvez faire comme ça [se penche], on peut voir que là dedans il y a une gros télé pour voir les films dans la machine. [...]

Marco: Au voilà, qu'est-ce qu'il y a là-bas près de la côte? Une grande maison jaune qui ressemble à un petit soleil. Ah! Oui! C'est mon auberge. Il s'appelle le 4 XXX et il avait cinq étoiles.

La description de Claudio montre qu'il conçoit son entreprise au-delà d'une dimension réaliste : cet hôtel serait un refuge de montagne où l'on accède avec sa Ferrari, voiture qu'il utilise pour sa démonstration, et que l'on peut même garer dans sa chambre! C'est pourquoi la créativité offre cet espace à l'imaginaire où l'on se soucie peu du matériel. La pensée divergente (Lubart) domine et chacun est libre de proposer ses solutions innovantes au monde hôtelier de demain. Un autre exemple, celui de Clara, élève du Piémont qui est venue suivre ses études secondaires en Vallée d'Aoste. Elle avait eu des cours intensifs pendant l'été, un voyage linguistique et un travail continu pendant toute l'année. Toutefois, à l'oral, elle rencontrait plus de difficultés à s'exprimer que ses pairs valdôtains. Le support créatif a constitué cette forme d'étayage (Bruner) qui lui a permis de s'exprimer plus facilement. La transcription suivante met en évidence la dimension structurée de sa production qui respecte le genre descriptif (nom du bar, description extérieure de 
l'établissement, puis description intérieure avec une précision du détail et l'utilisation d'un vocabulaire spécifique au genre).

Clara: Mon bar de mon rêve se trouve dans une rue centrale de la ville. Il s'appelle «musicien» et il y a non, il s'appelle "musicien ». L'aspect extérieur est ancien. Les couleurs est jaune clair, ma il y a des lignes courbes bordeau. Il y a une particulière fenêtre formée par l'entrelacement des figures géométriques qui est qui a l'air bizarre et particulière. À gauche, non à droite, il y a l'entrée. [L'élève ouvre l'une des parois du bar et l'on découvre un univers musical et coloré].

Élève : Madosca!

L'expression blasphématoire «Madosca! » traduit l'émerveillement d'un élève lorsque Clara a ouvert son support créatif. De même, lorsque Claudio a fait monter la voiture au drive-in, un élève s'est exclamé " Che figata! " ("c'est trop cool! ») et la plupart des élèves ont émis des commentaires d'émerveillement, certains ont aussi applaudi. S'il est difficile d'accéder aux émotions de l'élève-créateur sans réaliser d'auto-confrontation et uniquement par un processus d'inférence de la part de l'observateur, en revanche, il est plus évident de capter les émotions des élèves par leurs réactions qu'elles soient ici verbales ou non-verbales. Ces situations d'apprentissage permettent de mettre en évidence la nécessité de réfléchir sur l'émotion non seulement du point de vue de l'apprenant-créateur, mais aussi du point de vue des observateurs qui vivent une expérience vicariante.

\section{Conclusion}

Une pédagogie de la créativité s'interroge sur trois dimensions: émotionnelle, créative et cognitive. L'objectif est de proposer d'autres formes d'enseignement/apprentissage de la langue avec une réflexion théorique nécessaire pour mesurer le potentiel et les limites. Les enseignants mettent déjà en place, depuis plusieurs années, des activités ludiques pour varier les modalités d'enseignement. Toutefois, cet article a tenté de poser certaines réflexions théoriques qui permettent à la fois de légitimer au niveau scientifique des pratiques, mais surtout de conceptualiser une théorie qui peut enrichir, ou parfois changer, les pratiques. La créativité permet de "refonder l'acte d'enseignementapprentissage [...] en le structurant à partir de nouveaux concepts » (Aden, "Créativité », 179). D’autres pistes sont également à envisager dans une pédagogie de la créativité, notamment sur des recherches qui se font actuellement autour de l'empathie (Aden, «Empathie »). Elle permet aussi de réfléchir sur les difficultés d'apprentissage des langues, notamment durant une performance complexe. Une pédagogie de la créativité est un détour pour donner la possibilité au plus grand nombre possible d'élèves d'atteindre les mêmes 
objectifs. Enfin, elle propose des pistes d'action pour valoriser l'apprenant, sa compétence plurilingue et favoriser la construction d'une identité plurilingue.

\section{Bibliographie}

Aden, Joëlle. «La créativité artistique à l'école : refonder l'acte d'apprendre ». Synergies Europe 4 (2009) : 173-180.

---. «L'empathie, socle de la reliance en didactique des langues ». Enseigner les languescultures à l'ère de la complexité. Dir. J. Aden, T. Grimshaw et H. Penz. Bruxelles : Lang, 2010. 23-44.

--- et E. Piccardo, dirs. «La créativité dans tous ses états: enjeux et potentialités en éducation ». Synergies Europe 4 (2009).

Bandura, A. Auto-efficacité. Le sentiment d'efficacité personnelle. $2^{\mathrm{e}}$ éd. Tr. P. Lecomte. Bruxelles : De Boeck, 2007 [1997].

Barbier, R. La recherche action. Paris : Anthropos, 1996.

Beaud, S. et F. Weber. Guide de l'enquête de terrain. Paris : La Découverte, 2003.

Beaudot, A. Vers une pédagogie de la créativité. Paris : ESF, 1973.

Cambra Giné, M. Une approche ethnographique de la classe de langue. Paris : Didier, 2003.

Cavalli, M., A. Duchène, D. Elminger, et al. «Le bilinguisme : représentations sociales, discours et contextes ». Les représentations des langues et de leur apprentissage. Références, modèles, données et méthodes. Dir. D. Moore. Paris : Didier, 2007. 66-99.

Csikszentmihalyi, M. La créativité. Psychologie de la découverte et de l'invention. Tr. C-C. Farny. Trento / Paris : Erickson / Laffont, 2006 [1996].

Cuq, J.-P. Dictionnaire de didactique du français langue étrangère. Paris : Clé international, 2003.

Deforge, Y. L'œuvre et le produit. Seyssel : Champ Vallon, 1990.

Didier, J. et D. Leuba. «La conception d'un objet : un acte créatif ». Prismes 15 (2011) : 3233.

Gardner, H. Les cinq formes d'intelligence pour affronter l'avenir. Tr. M. Garène. Paris : Jacob, 2009 [2006].

Holley, A. «Créer avec des odeurs ». Les cinq sens de la création. Dir. M. Borillo \& A. Sauvageot. Seyssel : Champ Vallon, 1996. 25-28.

Laroche, S. «Un cerveau pour apprendre ». Apprendre et faire apprendre. Dir. É. Bourgeois et G. Chapelle. Paris : PUF, 2006. 39-52.

Lubart, T. Psychologie de la créativité. Paris : Armand Colin, 2003. 
Robinson, K. Out of Our Minds. Learning to Be Creative. West Sussex : Capstone, 2011.

Piccardo, E. Créativité et Technologies de l'Information et de la Communication dans l'enseignement/apprentissage des langues étrangères. Milano : Arcipelago, 2005.

--- et Isabelle Puozzo, dirs. «Émotion et apprentissage des langues ». Linguistique et Didactique des Langues (LIDIL), 2013 (à paraitre).

Puozzo Capron, Isabelle. «Français et pédagogie de la créativité ». L'école valdôtaine 91 (2012) : 61-63.

---. et R. Barioni. « Apprentissage autonome : du savoir à la compétence pragmatique ». Les Langues Modernes 3 (2012) : 55-61. 